Optical Society of America. Ronald J. Pieper, Jinwoo Park, and Ting-Chung Poon, "Resolution-dependent depth of focus for an incoherent imaging system," Appl. Opt. 27, 2040-2047 (1988). doi: 10.1364/AO.27.002040

\title{
Resolution-dependent depth of focus for an incoherent imaging system
}

\author{
Ronald J. Pieper, Jinwoo Park, and Ting-Chung Poon
}

\begin{abstract}
Through the application of the Rayleigh criterion for a two-point resolution, the dependence of the depth of focus on the resolution in the image is determined for a rectangular, a Gaussian, and an annular lens aperture. These solutions are posed in terms of the normalized parameters, obviating the need for the specification of the actual physical lengths. A general approach applicable to any aperture topology is discussed.
\end{abstract}

\section{Introduction}

The often cited criterion for the depth of focus is expressed solely in terms of the $f /$ No. of the lens and the wavelength of light. This resolution-independent depth of focus was derived ${ }^{1}$ by noting the effect of axial deviation relative to the in-focus plane at which the spatial frequency response begins to undergo accelerated deterioration. This condition was also obtained via a diffraction argument based on the uncertainty principle. ${ }^{2}$ Under the assumptions that the designer knows in advance the highest point density (resolution) required in the image, a more precise criterion can be formulated.

Although in the following discussion the Rayleigh criterion $^{3}$ for a two-point resolution was applied, other more stringent conditions ${ }^{4}$ could have been used. The approach we are taking in this discussion has been applied to specific apertures of current interest ${ }^{2,5-7}$; nevertheless, through numerical integration it should be applicable to any analytic form of transmittance aperture.

Starting from the block diagram representation of an imaging system, the incoherent impulse response is obtained. The resulting analytical expression is posed in a normalized form. The procedure for the application of the Rayleigh criterion to the normalized incoherent response is described for a general lens aperture topology. Resolution curves are plotted for a number of specific topologies.

The authors are with Virginia Polytechnic Institute \& State University, Bradley Department of Electrical Engineering, Blacksburg, Virginia 24061.

Received 15 July 1987.

0003-6935/88/102040-08\$02.00/0.

(C) 1988 Optical Society of America.
In Sec. II, the concepts motivating the analysis are discussed. In Sec. III, a general mathematical formalism is developed for any aperture function. In Sec. IV, three specific geometries for aperture transmittance are analyzed. In Sec. V, we present our conclusion that includes possible extensions of this work. In Appendix A, we start from the maximum realizable resolution for a Gaussian aperture and obtain, using the ray analysis, the correct dependence of the resolutionindependent depth of focus. In Appendix B, an example illustrating the use of the resolution curves is presented.

\section{General Description}

We assume that, consistent with the Fourier optics formalism, ${ }^{8}$ (1) the angles of diffraction are small, (2) the source is monochromatic but incoherent (i.e., the coherence length of the source is zero), and (3) the source, and therefore the complex field, has harmonic temporal dependence $\exp (-j \omega t)$ (physics convention for phasors).

The optical system to be analyzed is represented in Fig. 1. We also assume that the lens is aberration-free. Therefore, the defocus effect can be attributed solely to the axial deviation relative to the in-focus plane. The in-focus plane can be defined by setting $l^{\prime}=l_{f}^{\prime}$, where $l_{f}^{\prime}$ satisfies

$$
\frac{1}{l}+\frac{1}{l_{f}^{\prime}}=\frac{1}{f},
$$

and $f$ is the focal length of the lens. In general the outof-focus defect $\varepsilon$ is typically defined ${ }^{8}$ as

$$
\varepsilon=\frac{1}{f}-\frac{1}{l^{\prime}}-\frac{1}{l} \text {. }
$$

A point source of light, as shown in Fig. 1, can be treated mathematically as an impulse function. The impulse response is represented in the image plane a distance $l^{\prime}$ away from the lens. 


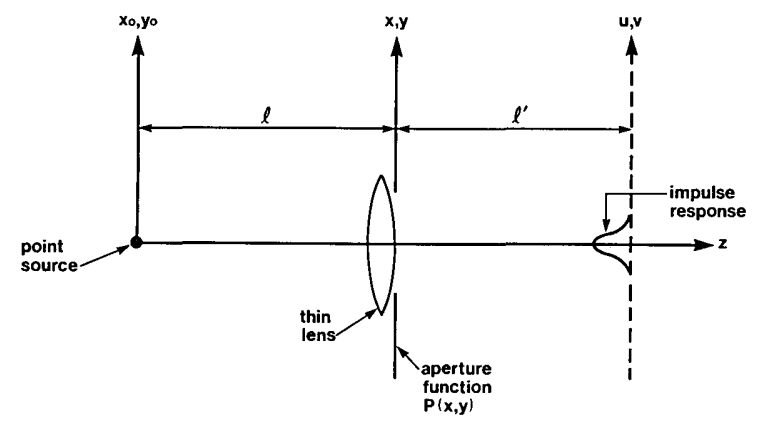

Fig. 1. Optical configuration examined for determination of the incoherent impulse response.

In Fig. 2, the response for two-point impulses is shown, and according to the Rayleigh criterion they can be resolved if

$$
d \geq W \text {, }
$$

where $W$ is the full width at half-maximum (FWHM) intensity of the incoherent impulse response, and $d$ is the center-to-center spacing.

Imagine that in the object plane a 1-D array of point sources exists as represented in Fig. 3. The spatial density of points $\sigma$ generated in the image plane is, assuming unity magnification, given by

$$
\sigma=1 / d \text {. }
$$

Therefore, the maximum point density $\rho$ that can be resolved, according to the Rayleigh criterion, is

$$
\rho=1 / W \geq \sigma .
$$

In the remainder of the text we will refer to this parameter as the resolvable point density. Once $W$ is related to the defocus defect $\varepsilon$, the relation between the resolvable point density and the defocus defect follows from expression (5). To determine $W$, the incoherent impulse response has to be determined. This is obtained from the magnitude squared of the complex coherent impulse response. ${ }^{8}$ It is apparent that the geometrical scale factor associated with the magnification in the image has not been incorporated in this discussion. Since the magnification scale factor $M$ equally affects both sides of inequality (3), a unity magnification can be assumed without loss in generality.

\section{Analysis}

Under the assumptions stated in the previous section, the incoherent impulse response can be determined through the application of a block diagram system approach, ${ }^{8}$ as shown in Fig. 4. The propagation impulse responses are given by

$$
\begin{aligned}
& h_{l}=C_{1} \exp \left[j \frac{k}{2 l}\left(x^{2}+y^{2}\right)\right], \\
& h_{l^{\prime}}=C_{2} \exp \left[j \frac{k}{2 l^{\prime}}\left(x^{2}+y^{2}\right)\right],
\end{aligned}
$$

where $k=2 \pi / \lambda$ is the propagation constant in air, $C_{1}$ and $C_{2}$ are complex constants, and $x$ and $y$ are the Cartesian coordinates as defined in Fig. 1. The ideal

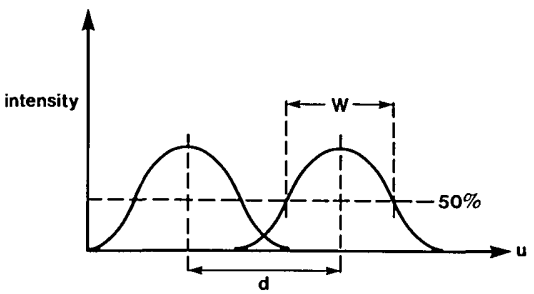

Fig. 2. Two impulse responses with defining parameters for the Rayleigh criterion.

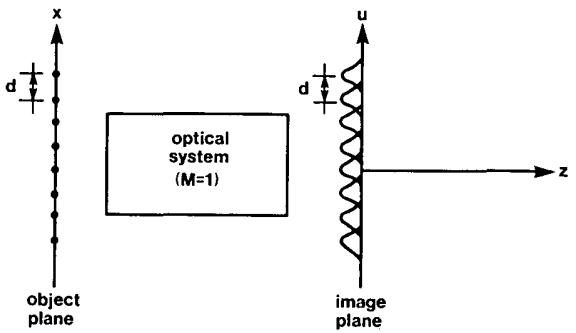

Fig. 3. Optical system response to spatially periodic impulses.

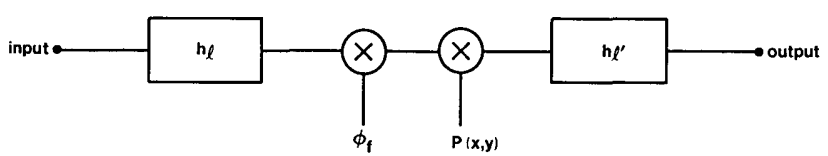

Fig. 4. System model for the optical system in Fig. 1 .

thin lens of focal length $f$ introduces a spatially quadratic phase factor $\phi_{f}$ given by

$$
\phi_{f}=\exp \left[-j \frac{k}{2 f}\left(x^{2}+y^{2}\right)\right] .
$$

The coherent impulse response of the entire optical system $h_{s}$ is then expressible in terms of expressions (6):

$$
h_{s}=\left[h_{l} \phi_{f} P(x, y)\right] * h_{l^{\prime}},
$$

where $*$ denotes 2 -D spatial convolution and $P(x, y)$ is the aperture transmittance placed against the lens. Using definition (2), coherent response (7) can be expressed explicitly as

$$
\begin{aligned}
h_{s}(u, v)= & \exp \left[j \frac{k}{2 l^{\prime}}\left(u^{2}+v^{2}\right)\right] \int_{-\infty}^{\infty} \int_{-\infty}^{\infty} \exp \left[-j \frac{k}{2} \varepsilon\left(x^{2}+y^{2}\right)\right] P(x, y) \\
& \times \exp \left[-j \frac{k}{2 l^{\prime}}(2 u x+2 v y)\right] d x d y
\end{aligned}
$$

where the complex constant term has been dropped, and the Cartesian coordinates $u, v$ are defined in Fig. 1. Since the incoherent impulse response is obtained from the magnitude squared of the coherent impulse response, the complex exponential term in Eq. (8), which precedes the integration, will not make any contribution and will, therefore, be dropped.

The result in Eq. (8) can be written in a compact form as 


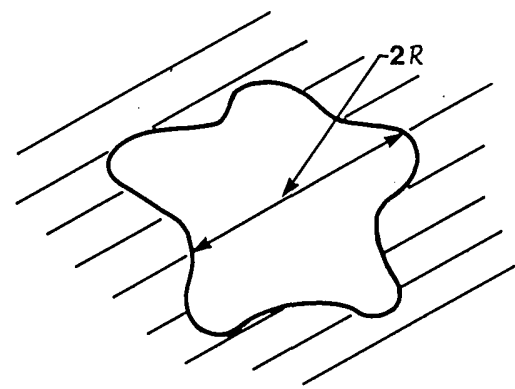

Fig. 5. General aperture function with a typical scale dimension $\mathcal{R}$.

$$
h_{s}(u, v)=F\left\{\exp \left[-j \frac{k}{2} \varepsilon\left(x^{2}+y^{2}\right)\right] P(x, y)\right\} \mid \begin{aligned}
& f_{x}=u / \lambda l^{\prime} \\
& f_{y}=v / \lambda l^{\prime}
\end{aligned},
$$

where the Fourier transform operation, $F\{\cdot\}$, is defined on an arbitrary function $t(x, y)$ as

$$
F\{t(x, y)\} \equiv \int_{-\infty}^{\infty} \int_{-\infty}^{\infty} t(x, y) \exp \left[-j 2 \pi\left(f_{x} x+f_{y} y\right)\right] d x d y,
$$

with $f_{x}$ and $f_{y}$ denoting the spatial frequencies.

We define the integrated transmittance, $A(P)$, of the aperture as

$$
A(P)=\int_{-\infty}^{\infty} \int_{-\infty}^{\infty} P(x, y) d x d y .
$$

In all physically meaningful applications, some nonunique geometric scale, $\mathcal{R}$, proportional to the square root of the integrated transmittance can be defined. This is represented in Fig. 5. The new normalized spatial variables $x^{\prime}$ and $y^{\prime}$ are defined as

$$
\begin{aligned}
& x^{\prime}=x / \mathcal{R}, \\
& y^{\prime}=y / \mathcal{R} .
\end{aligned}
$$

A scale-independent aperture transmittance $p\left(x^{\prime}, y^{\prime}\right)$ can then be defined:

$$
p\left(x^{\prime}, y^{\prime}\right) \equiv P\left(\mathcal{R} x^{\prime}, \mathcal{R} y^{\prime}\right) .
$$

It follows, after some algebra, that the coherent impulse response in Eq. (9) can be written as

$$
\begin{aligned}
\frac{h_{s}\left(\alpha^{\prime}, \alpha^{\prime \prime}, \beta\right)}{A(P)}= & \frac{1}{A(p)} \int_{-\infty}^{\infty} \int_{-\infty}^{\infty} \exp \left[-j \frac{\pi}{2} \beta^{2}\left(x^{\prime 2}+y^{\prime 2}\right)\right] \\
& \times \exp \left[-j \pi\left(\alpha^{\prime} x^{\prime}+\alpha^{\prime \prime} y^{\prime \prime}\right)\right] p\left(x^{\prime}, y^{\prime}\right) d x^{\prime} d y^{\prime}
\end{aligned}
$$

where

$$
\begin{aligned}
A(p) & \equiv \int_{-\infty}^{\infty} \int_{-\infty}^{\infty} p\left(x^{\prime}, y^{\prime}\right) d x^{\prime} d y^{\prime} \equiv \frac{1}{\mathcal{R}^{2}} \int_{-\infty}^{\infty} \int_{-\infty}^{\infty} P(x, y) d x d y, \\
\beta & \equiv \sqrt{\frac{k \varepsilon}{\pi}} \mathcal{R}, \\
\alpha^{\prime} & \equiv k u \mathcal{R} / \pi l^{\prime}, \\
\alpha^{\prime \prime} & \equiv k v \mathcal{R} / \pi l^{\prime} .
\end{aligned}
$$

It can be noted that transmittance (14b) does not depend on the physical scale of the aperture. The defect parameter $\beta$ is independent of the transverse coordinates $u, v$. Finally, the normalized transverse coordinates $\alpha^{\prime}$ and $\alpha^{\prime \prime}$ are not dependent on the out-of-

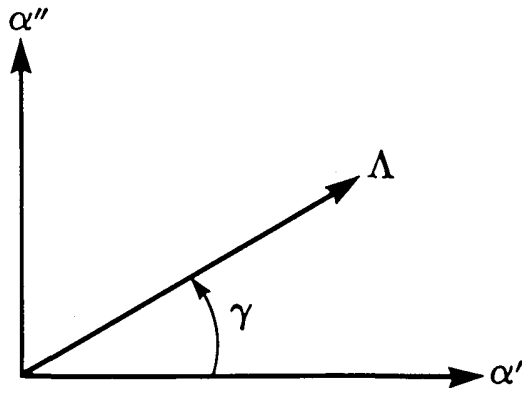

Fig. 6. Defining relationship between $(\Lambda, \gamma)$ and $\left(\alpha^{\prime}, \alpha^{\prime \prime}\right)$.

focus defect $\varepsilon$. Therefore, the normalization obviates the specification of physical lengths, e.g., $l, l^{\prime}, \mathcal{R}, f$, and $\lambda$ in the evaluation of Eq. (14a). It is apparent that for $\alpha^{\prime}=0, \alpha^{\prime \prime}=0$, and $\beta=0$ (i.e., on-axis and in-focus) the right-hand side of Eq. (14a) is unity. Expression (14a) has, therefore, been normalized to the maximum magnitude.

Using definition (10), the normalized coherent impulse response (14a) can be written as

$\frac{h_{s}\left(\alpha^{\prime}, \alpha^{\prime \prime}, \beta\right)}{A(P)}=\left.\frac{1}{A(p)} F\left\{\exp \left[-j \frac{\pi}{2} \beta^{2}\left(x^{2}+y^{\prime 2}\right)\right] p\left(x^{\prime}, y^{\prime}\right)\right\}\right|_{f_{y}=\frac{\alpha^{\prime \prime}}{2}} ^{f_{x}=\frac{\alpha^{\prime}}{2}}$.

If the function $p\left(x^{\prime}, y^{\prime}\right)$ has circular symmetry, the transform above can be written equivalently as a Fourier Bessel transform $B\{\}\}^{8}$ as

$$
\begin{array}{r}
\frac{h_{s}\left(\alpha^{\prime}, \alpha^{\prime \prime}, \beta\right)}{A(P)}=\frac{1}{A(p)} B\left\{\exp \left(-j \frac{\pi}{2} \beta^{2} r^{\prime 2}\right) p\left(r^{\prime}\right)\right\} \mid \\
\rho=\sqrt{\left(\frac{\alpha^{\prime}}{2}\right)^{2}+\left(\frac{\alpha^{\prime \prime}}{2}\right)^{2}},
\end{array}
$$

where the Bessel transform is defined in terms of the zeroth-order Bessel function of the first kind:

$$
B\left\{t\left(r^{\prime}\right)\right\} \equiv 2 \pi \int_{0}^{\infty} r^{\prime} J_{0}\left(2 \pi r^{\prime} \rho\right) t\left(r^{\prime}\right) d r^{\prime} .
$$

The normalized incoherent response is obtained from the square of the magnitude of the normalized coherent response. Note that the FWHM width of the normalized incoherent impulse response will not be the same in all directions in the image plane, assuming that the aperture does not possess polar symmetry. Therefore, the orientation for minimum resolvable line density is, according to the Rayleigh criterion, the direction in which the incoherent impulse response exhibits the maximum width.

Let us assume that the relation between the depth of focus and the resolvable point density have to be determined for a particular orientation of points. Figure 6 defines the parameters $\alpha^{\prime}$ and $\alpha^{\prime \prime}$ in terms of a normalized length $\Lambda$ and an angle $\gamma$ :

$$
\begin{aligned}
\alpha^{\prime} & =\Lambda \cos (\gamma), \\
\alpha^{\prime \prime} & =\Lambda \sin (\gamma) .
\end{aligned}
$$

With the introduction of the parameters $\Lambda$ and $\gamma$, the incoherent response can be interrogated for any direc- 
tion in the $\alpha^{\prime}-\alpha^{\prime \prime}$ plane. A normalized FWHM width, $\Delta \Lambda$, along direction $\gamma$, can be defined, following (14d) and $(14 \mathrm{e})$, as

$$
\Delta \Lambda \equiv\left(\frac{k \mathscr{R}}{\pi l^{\prime}}\right) W .
$$

The normalized width $\Delta \Lambda$ will be determined from the examination of the normalized incoherent impulse response $\left|h_{s}\left(\alpha^{\prime}, \alpha^{\prime \prime}, \beta\right) / A(P)\right|^{2}$, subject to constraint (17) for various $\beta$. The curves which illustrate the expected behavior of $\Delta \Lambda$ as a function of $\beta$ are shown in Fig. 7(a). The implicit dependence of $\Delta \Lambda$ on the out-offocus defect parameter $\beta$ has been emphasized. By combining Eqs. (5) and (18), it is clear that the resolvable point density satisfies

$$
\rho(\beta)=\frac{1}{W}=\left[\frac{k \mathscr{R}}{\pi l^{\prime}}\right]\left[\frac{1}{\Delta \Lambda}\right] .
$$

Again, the implicit dependence of $\rho$ on $\beta$ follows from Fig. 7(a). Based on the examination of Fig. 7(a), a plot of $(1 / \Delta \Lambda)$ vs the defect parameter $\beta$ is sketched in Fig. 7(b). It follows from expression (19), after referring to definition (14c), that the resolvable point density $\rho$ as a function of focus defect $\varepsilon$ can be determined. An example of this type of calculation is presented in Appendix B. In the next section this analysis is applied to some specific geometries.

\section{Specific Geometries}

\section{A. Rectangular Aperture}

The rectangular geometry aperture, represented in Fig. 8(a), can be expressed as

$$
P(x, y)=\operatorname{rect}\left(x / L_{x}\right) \operatorname{rect}\left(y / L_{y}\right)
$$

where

$$
\begin{aligned}
\operatorname{rect}(\xi) & =1 \text { for }|\xi| \leq 1 / 2 \\
& =0 \text { for }|\xi| \geq 1 / 2 .
\end{aligned}
$$

If we take $\mathcal{R}=\left(\sqrt{L_{x} L_{y}}\right) / 2$ and define $s \equiv \sqrt{\left(L_{x} / L_{y}\right)}$, the scale-independent aperture transmittance becomes

$$
p\left(x^{\prime}, y^{\prime}\right)=\operatorname{rect}\left(\frac{x^{\prime}}{2 s}\right) \operatorname{rect}\left(\frac{y^{\prime} s}{2}\right)
$$

And it follows from definitions (14b)-(14e) that

$$
\begin{aligned}
A(p) & =\int_{-1 / s}^{1 / s} \int_{-s}^{s} d x^{\prime} d y^{\prime}=4, \\
\beta & =\sqrt{\frac{k_{\varepsilon}}{\pi}} \frac{\sqrt{L_{x} L_{y}}}{2}, \\
\alpha^{\prime} & =\frac{k u}{\pi l^{\prime}} \frac{\sqrt{L_{x} L_{y}}}{2}, \\
\alpha^{\prime \prime} & =\frac{k v}{\pi l^{\prime}} \frac{\sqrt{L_{x} L_{y}}}{2} .
\end{aligned}
$$

For this geometry, the $x^{\prime}$ and $y^{\prime}$ integrations (14a) are uncoupled and of essentially the same functional form. After completing the square and dropping an inessential phase factor, the normalized coherent impulse response in (14a) can be written as
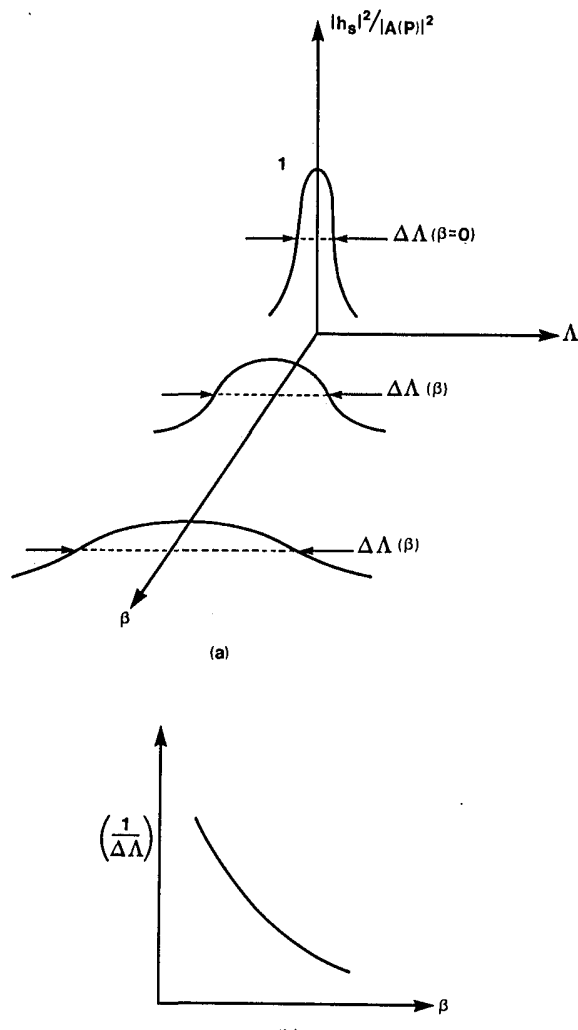

Fig. 7. Sketches based on physical interpretation of Eqs. (16)-(19): (a) typical normalized impulse response curves; (b) resolvable point density vs the defect parameter $\beta$.

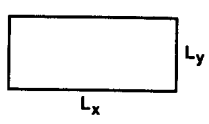

(a)

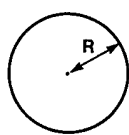

(b)

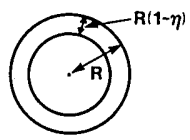

(c)
Fig. 8. Specific geometries analyzed in Sec. IV: (a) rectangular; (b) Gaussian ( $R=$ Gaussian width at 1/e of maximum); (c) annular.

$$
\begin{aligned}
\frac{h_{s}\left(\alpha^{\prime}, \alpha^{\prime \prime}, \beta\right)}{A(P)}= & \frac{1}{4} \int_{-s}^{s} \exp \left[-j \frac{\pi}{2} \beta^{2}\left(x^{\prime}+\frac{\alpha^{\prime}}{\beta^{2}}\right)^{2}\right] d x^{\prime} \int_{-1 / s}^{1 / s} \\
& \times \exp \left[-j \frac{\pi}{2} \beta^{2}\left(y^{\prime}+\frac{\alpha^{\prime \prime}}{\beta^{2}}\right)^{2}\right] d y^{\prime} .
\end{aligned}
$$

For the purpose of compactly expressing the normalized incoherent impulse response, the following combinations of Fresnel integrals are defined:

$$
\begin{aligned}
f\left(\alpha^{\prime}, \beta\right)= & \frac{1}{4 \beta^{2}}\left\{\left[C\left(\frac{\alpha^{\prime}}{\beta}+\beta s\right)-C\left(\frac{\alpha^{\prime}}{\beta}-\beta s\right)\right]^{2}\right. \\
& \left.+\left[S\left(\frac{\alpha^{\prime}}{\beta}+\beta s\right)-S\left(\frac{\alpha^{\prime}}{\beta}-\beta s\right)\right]^{2}\right\}, \\
g\left(\alpha^{\prime \prime}, \beta\right)= & \frac{1}{4 \beta^{2}}\left\{\left[C\left(\frac{\alpha^{\prime \prime}}{\beta}+\frac{\beta}{s}\right)-C\left(\frac{\alpha^{\prime \prime}}{\beta}-\frac{\beta}{s}\right)\right]^{2}\right. \\
& \left.+\left[S\left(\frac{\alpha^{\prime \prime}}{\beta}+\frac{\beta}{s}\right)-S\left(\frac{\alpha^{\prime \prime}}{\beta}-\frac{\beta}{s}\right)\right]^{2}\right\},
\end{aligned}
$$

where 


$$
\begin{aligned}
& C(\xi) \equiv \int_{0}^{\xi} \cos \left(\frac{\pi}{2} \tau^{2}\right) d \tau, \\
& S(\xi) \equiv \int_{0}^{\xi} \sin \left(\frac{\pi}{2} \tau^{2}\right) d \tau .
\end{aligned}
$$

Using the definitions in (24), the normalized incoherent impulse response becomes

$$
\left|\frac{h_{s}\left(\alpha^{\prime}, \alpha^{\prime \prime}, \beta\right)}{A(P)}\right|^{2}=f\left(\alpha^{\prime}, \beta\right) g\left(\alpha^{\prime \prime}, \beta\right) .
$$

To find the normalized FWHM width, it is necessary to examine the dependence of the response in a specific direction. For illustration the $s$ parameter was arbitrarily set to 2.0. The two cases, which will be tested, correspond to $\gamma=0$ and $\gamma=\pi / 2$. The conditions on the impulse response given by Eq. (25) become

$$
\begin{aligned}
& \Lambda=\alpha^{\prime}, \alpha^{\prime \prime}=0, \quad s=2.0, \\
& \Lambda=\alpha^{\prime \prime}, \alpha^{\prime}=0, \quad s=2.0,
\end{aligned}
$$

respectively. Representative normalized impulse response curves, subject to conditions (26a) and (26b), have been plotted in Fig. 9 for defect parameters $\beta=$ $0.0,0.5,0.7,1.1$. Although (24) cannot be explicitly evaluated for $\beta=0.0$, the graphical results were found to converge for small enough $\beta$, e.g., $\beta<0.05$. It can be confirmed that the graphic solution, obtained in this manner, agrees with the results obtained analytically from Eq. (15) after setting $\beta=0.0$. From curves such as those shown in Fig. 9(a), a plot of the reciprocal of the normalized FWHM, $1 / \Delta \alpha^{\prime}$, vs the defect parameter $\beta$ is obtained. This is shown as the upper curve in Fig. 10. This curve can be directly related to the resolvable point density [see Eq. (19)] since, in the case of (19) or $\gamma$ $=0.0$, it follows from Eq. (26a) that

$$
\Delta \Lambda=\Delta \alpha^{\prime} .
$$

Similarly, from Eq. (26b), we have

$$
\Delta \Lambda=\Delta \alpha^{\prime \prime} .
$$

For this case the reciprocal of the normalized FWHM, $1 /\left(\Delta \alpha^{\prime \prime}\right)$, is shown as the lower curve in Fig. 10 . As expected from the direct evaluation of Eq. (14a), after taking $\beta=0.0$, the results, represented in Fig. 10, indicate the in-focus diffraction FWHM along the coordinate $\alpha^{\prime}$ [condition (26a)] is $1 / 4$ of the diffraction FWHM along the coordinate $\alpha^{\prime \prime}$ [condition (26b)]. In addition, the resolution in condition (26a), i.e., the upper curve; deteriorates faster with the defocus defect parameter $\beta$ than that in condition (26b) (lower curve). This is qualitatively understood by employing the circle-of-confusion argument ${ }^{3}$ which predicts more rapid deterioration in the resolution for apertures with larger dimensions.

\section{B. Gaussian Aperture Function}

The Gaussian aperture function, represented in Fig. $8(\mathrm{~b})$, is given by.

$$
P(x, y) \equiv \exp \left(-r^{2} / R^{2}\right)
$$

If we take the normalizing dimension to be $R=R$,
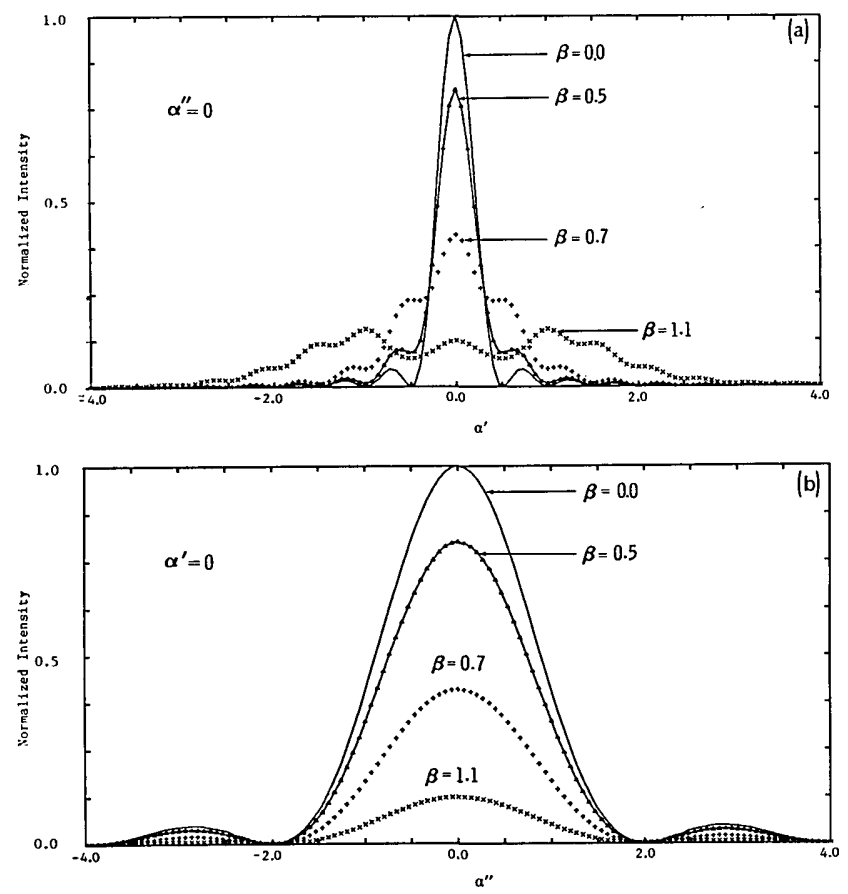

Fig. 9. Impulse response curve for rectangular geometry with $s=2$ (i.e., $L_{x}=4 L_{y}$ ): (a) along coordinate $\alpha^{\prime}$; (b) along coordinate $\alpha^{\prime \prime}$.

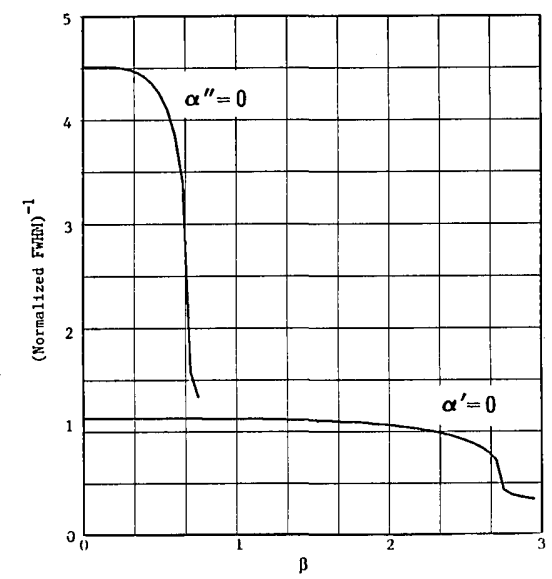

Fig. 10. Resolution curves for rectangular geometry: upper curve is $1 /$ FWHM along direction $\alpha^{\prime}$; lower curve is $1 /$ FWHM along direction $\alpha^{\prime \prime}$.

$$
\begin{aligned}
p\left(x^{\prime}, y^{\prime}\right) & =\exp \left[-\left(r^{\prime}\right)^{2}\right], \\
A(p) & =2 \pi \int_{0}^{\infty} \exp \left[-\left(r^{\prime}\right)^{2}\right] \times r^{\prime} d r^{\prime}=\pi .
\end{aligned}
$$

The normalized coherent impulse response in Eq. (15) can then be expressed as

$$
\frac{h_{s}\left(\alpha^{\prime}, \alpha^{\prime \prime}, \beta\right)}{A(P)}=\left.\frac{1}{\pi} F\left\{\exp \left[-\left(j \frac{\pi}{2} \beta^{2}+1\right)\left(x^{\prime 2}+y^{\prime 2}\right)\right]\right\}\right|_{f_{y}=\frac{\alpha^{\prime \prime}}{2}} ^{f_{x}=\frac{\alpha^{\prime}}{2} \times},
$$

where $\alpha^{\prime}, \alpha^{\prime \prime}, \beta$ are given, after substituting $R$ for $\mathcal{R}$, by $(14 \mathrm{c}),(14 \mathrm{~d})$, and $(14 \mathrm{e})$, respectively. Using a transform table, Eq. (30) can be reduced to 


$$
\frac{h_{s}\left(\alpha^{\prime}, \alpha^{\prime \prime}, \beta\right)}{A(P)}=\left.\frac{1}{|a|} \exp \left[-\frac{\pi^{2}}{a}\left(f_{x}^{2}+f_{y}^{2}\right)\right]\right|_{f_{y}=\frac{\alpha^{\prime \prime}}{2}} ^{f_{x}=\frac{\alpha^{\prime}}{2}},
$$

where for convenience of notation we define

$$
a \equiv j \frac{\pi}{2} \beta^{2}+1 .
$$

Due to the polar symmetry, $\alpha^{\prime \prime}$ can be set to zero without loss in generality. The incoherent impulse response then becomes

$$
\left|\frac{h_{s}\left(\alpha^{\prime}, 0, \beta\right)}{A(P)}\right|^{2}=\frac{1}{1+\left(\frac{\pi}{2} \beta^{2}\right)^{2}} \exp \left\{-2 \pi^{2} \alpha^{2} /\left[4+\pi\left(\beta^{2}\right)^{2}\right]\right\} .
$$

These curves, for arbitrary $\beta$, drop to half of the maximum of the impulse response at a value for $\alpha^{\prime}$ satisfying

$$
\begin{aligned}
\alpha^{\prime} & =\alpha_{1 / 2}^{\prime} \\
& \equiv \frac{1}{\pi} \sqrt{\frac{\ln 2}{2}\left[4+\left(\pi \beta^{2}\right)^{2}\right] .}
\end{aligned}
$$

It follows that $1 /(\Delta \Lambda)$ can be expressed in terms of $\beta$ as

$$
\frac{1}{\Delta \Lambda}=\frac{1}{\Delta \alpha^{\prime}} \equiv \frac{1}{2\left(\alpha_{1 / 2}^{\prime}\right)}=\frac{\pi}{2}\left\{\frac{\ln 2}{2}\left[4+\left(\pi \beta^{2}\right)^{2}\right]\right\}^{-1 / 2}
$$

This dependence is plotted in Fig. 11. In Appendix A we show how this result of Eq. (34), combined with the ray theory, leads to, up to a constant, the well-known resolution-independent form for the depth of focus.

\section{Annular Aperture Function}

The aperture function, represented in Fig. 8(c), can be analytically expressed as

$$
P(x, y)=\operatorname{circ}(r / R)-\operatorname{circ}(r / \eta R),
$$

where

$$
\operatorname{circ}(r / D)=\begin{aligned}
& 1 \text { for } r<D \\
& 0 \text { for } r>D
\end{aligned}
$$

and the annular factor $\eta$ satisfies

$$
1 \geq \eta \geq 0 \text {. }
$$

Taking the scale dimension $\mathcal{R}$ equal to $R$ leads to

$$
\begin{aligned}
& p\left(r^{\prime}\right)=\operatorname{circ}\left(r^{\prime}\right)-\operatorname{circ}\left(r^{\prime} / \eta\right), \\
& A(p)=\pi\left(1-\eta^{2}\right) .
\end{aligned}
$$

In this case the normalized coherent impulse response, as derived from expression (16), can be written as

$$
\begin{array}{r}
\frac{h_{s}\left(\alpha^{\prime}, \alpha^{\prime \prime}, \beta\right)}{A(P)}=\frac{1}{\pi\left(1-\eta^{2}\right)} B\left\{-j \frac{\pi}{2} \beta^{2} r^{\prime 2} p\left(r^{\prime}\right)\right\} \mid \\
\rho=\sqrt{\left(\frac{\alpha^{\prime}}{2}\right)^{2}+\left(\frac{\alpha^{\prime \prime}}{2}\right)^{2}} .
\end{array}
$$

After setting $\alpha^{\prime \prime}=0$, Eq. (37) can be expressed, using definition (16a), as

$$
\left|\frac{h_{s}\left(\alpha^{\prime}, 0, \beta\right)}{A(P)}\right|^{2}=\left[\frac{2}{\left(1-\eta^{2}\right)}\right]^{2}\left|\int_{\eta}^{1} r^{\prime} J_{0}\left(\pi \alpha^{\prime} r^{\prime}\right) \exp \left(-j \frac{\pi}{2} \beta^{2} r^{\prime 2}\right) d r^{\prime}\right|^{2} .
$$

Normalized impulse response $\beta=0.0,1.1,1.6,1.9$ $\left(\frac{1}{\Delta \Lambda}\right)_{\text {vs } \beta}$

\begin{tabular}{lll}
\hline$\eta=0.0$ & Fig. 12 & Fig. 17 \\
$\eta=0.3$ & Fig. 13 & Fig. 17 \\
$\eta=0.6$ & Fig. 14 & Fig. 17 \\
$\eta=0.9$ & Fig. 15 & Fig. 17 \\
$\eta=0.99$ & Fig. 16 & Fig. 17 \\
\hline
\end{tabular}

For the purpose of numerical calculation, the above can be recast in the following strictly real form:

$$
\left|\frac{h_{s}\left(\alpha^{\prime}, 0, \beta\right)}{A(P)}\right|^{2}=\left[\frac{2}{\left(1-\eta^{2}\right)}\right]^{2}\left(I_{1}^{2}+I_{2}^{2}\right)
$$

where

$$
\begin{aligned}
& I_{1}=\int_{\eta}^{1} r^{\prime} J_{0}\left(\pi \alpha^{\prime} r^{\prime}\right) \cos \left(\frac{\pi}{2} \beta^{2} r^{2}\right) d r^{\prime}, \\
& I_{2}=\int_{\eta}^{1} r^{\prime} J_{0}\left(\pi \alpha^{\prime} r^{\prime}\right) \sin \left(\frac{\pi}{2} \beta^{2} r^{\prime 2}\right) d r^{\prime} .
\end{aligned}
$$

Table I lists the curves generated based on Eqs. (39). Note that, for $\alpha^{\prime \prime}=0$, relation (27a) applies. The normalized incoherent impulse response curves correspond to $\eta=0.0,0.3,0.6,0.9$, and 0.99 are shown in Figs. $12-16$, respectively. We note a decrease in sensitivity to the defect parameter $\beta$ with increasing $\eta$, in agreement with predictions from previous investigations. ${ }^{2,5}$ The resulting resolution curves, as presented in Fig. 17, summarize this observation.

\section{v. Conclusion}

A formalism was introduced for expressing the incoherent impulse response in terms of the normalized parameters. Because of this normalization the analysis only requires the specification of the aperture topology, independent of any physical lengths. By using the Rayleigh criterion for a two-point resolution, the depth of focus was related to the level of resolution required in the image. In the case of a Gaussian aperture, this process was analytically tractable. These results were shown to lead to, up to a constant, the resolution-independent form for the depth of focus.

The extension of this work to nonimpulse type objects, i.e., input waveforms such as the sinusoids and the square wave profiles, is presently being studied.

This work was supported in part by the Fiber \& Electro-Optics Research Center, Virginia Polytechnic Institute \& State University. The authors wish to thank L. Hsu-Pieper for assistance in the final preparation of the manuscript.

\section{Appendix A. Heuristic Derivation of the Resolution- Independent Depth of Focus}

In this Appendix, it is shown that, up to a constant, the well-known resolution-independent depth of focus $^{2}$ follows from the analytic expression (31) in the worst-case conditions. Specifically, we will examine 


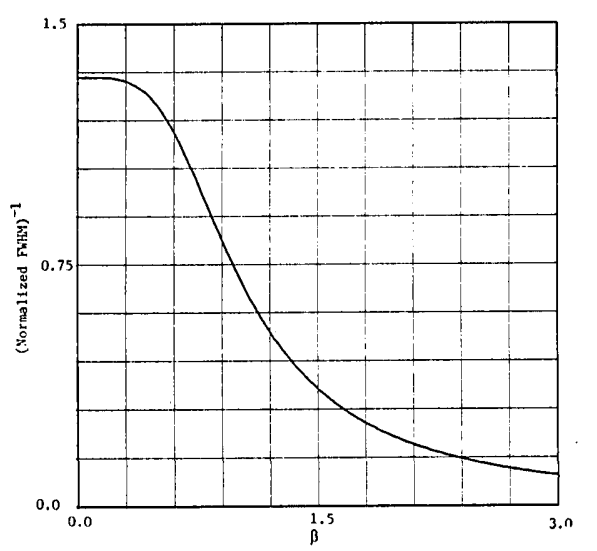

Fig. 11. Resolution curve for Gaussian aperture.

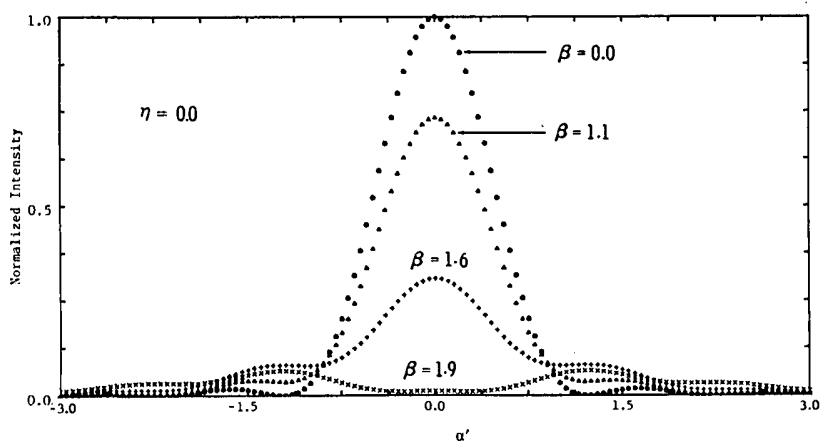

Fig. 12. Impulse response curves for annular aperture $\eta=0.0$.

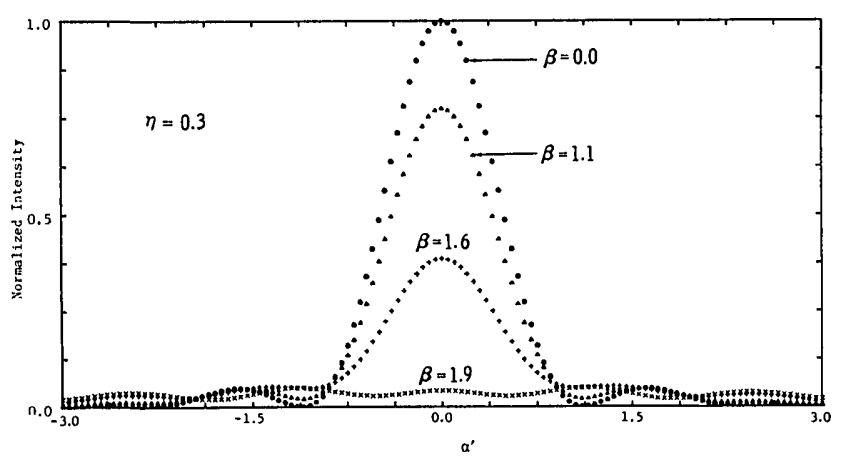

Fig. 13. Impulse response curves for annular aperture $\eta=0.3$.

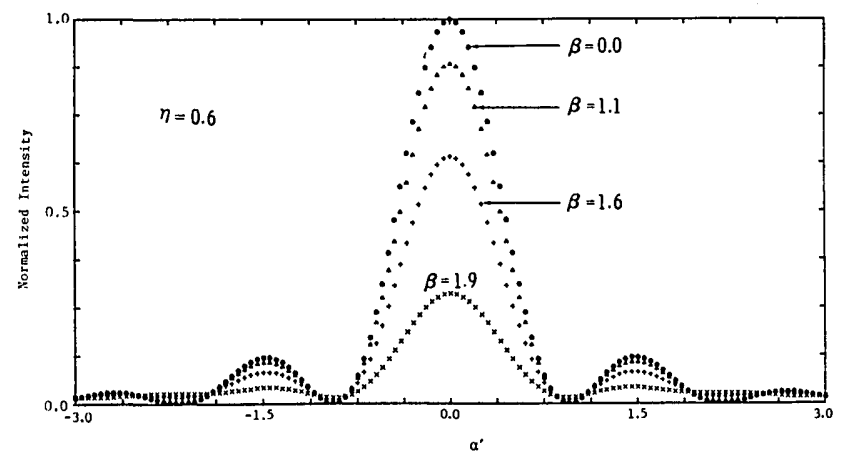

Fig. 14. Impulse response curves for annular aperture $\eta=0.6$.

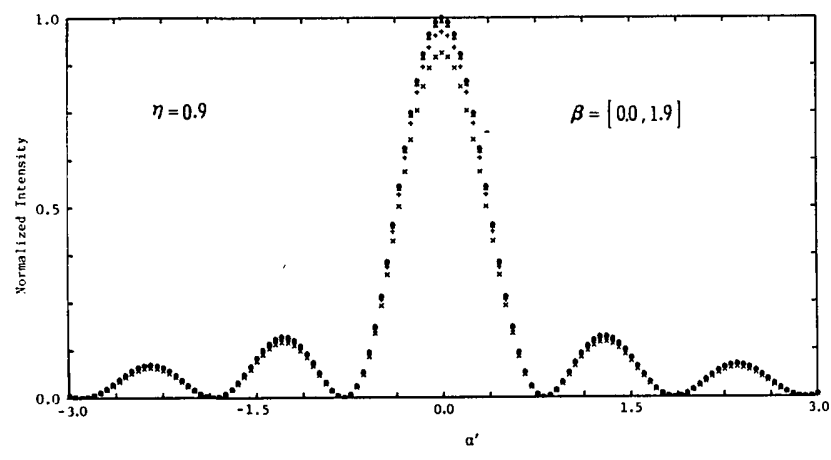

Fig. 15. Impulse response curves for annular aperture $\eta=0.9$.

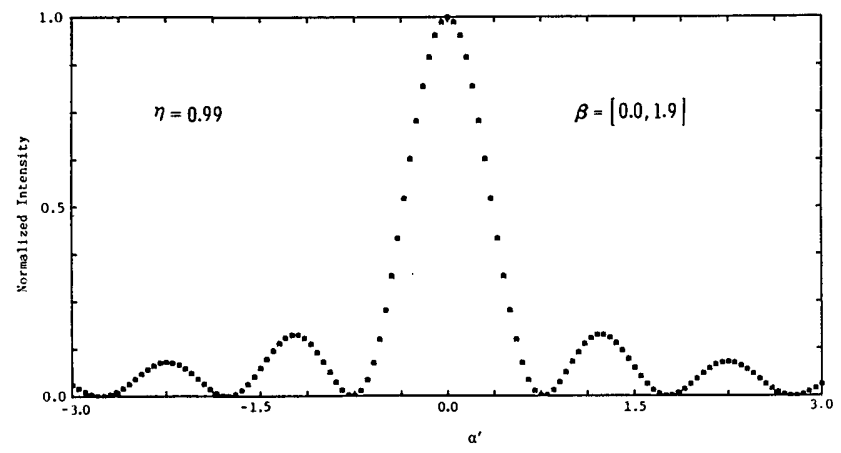

Fig. 16. Impulse response curves for annular aperture $\eta=0.99$.

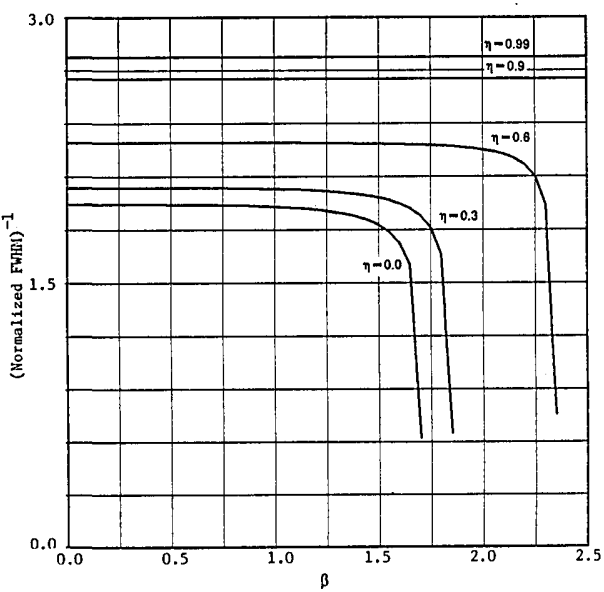

Fig. 17. Resolution curves for the annular apertures described in Table I.

the depth-of-focus condition, using a ray optics interpretation of the Rayleigh criterion for the maximum resolvable point density permitted by the impulse response, which, by inspection, occurs for $\beta=0.0$. From Eqs. (19) and (34) it follows that

$$
\begin{aligned}
\rho(0) & =\frac{\pi}{\sqrt{2 \ln 2}} \frac{R}{\lambda l^{\prime}} \\
& \cong 2.67 \frac{R}{\lambda l^{\prime}} .
\end{aligned}
$$

This can be compared with the highest allowed spatial frequency for the circular pupil function, ${ }^{8}$ 


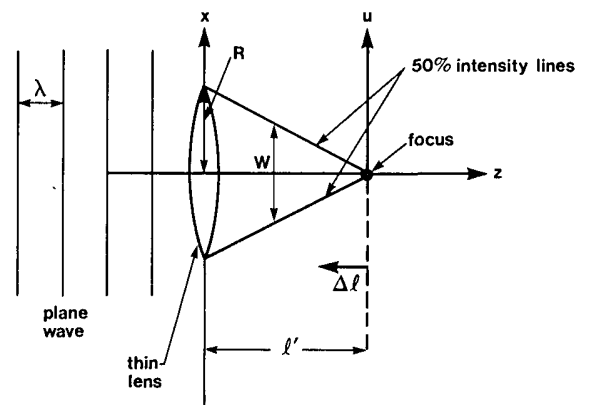

Fig. 18. Ray model representing the converging action of a lens with $50 \%$ intensity lines.

$$
\rho(0)=2 \frac{R}{\lambda l^{\prime}} .
$$

Figure 18 is a ray model representation for the converging action of a lens on a plane wave. $W$ represents the vertical width between $50 \%$ intensity lines at a horizontal location $\Delta l$ to the left of focus. For small angles, $W$ can be expressed as

$$
W=2 \Delta l\left(R / l^{\prime}\right) \text {. }
$$

Following the concepts presented in Sec. II,

$$
\rho(\beta)=\frac{1}{W} \leq \rho(0) .
$$

Combining (3) and (4), and employing the equality in Eq. (A4) will lead to a maximum allowed deviation:

$$
\Delta l=\frac{l^{\prime}}{2 R \rho(0)}
$$

After the final substitution from Eq. (A1), Eq. (A5) becomes

$$
\Delta l \cong 0.75\left(l^{\prime} / 2 R\right)^{2} \lambda \geq 0.75(f / 2 R)^{2} \lambda .
$$

Taking the worst case for $\Delta l$ by setting $l^{\prime}=f$ and employing the definition for the $f /$ No. of the lens, Eq. (A6) can be written as

$$
\Delta l=0.75(f / \mathrm{No} .)^{2} \lambda .
$$

This agrees with the results cited in Refs. 1 and 2 up to a multiplicative constant factor.

\section{Appendix B. Example Calculation for the Depth of Focus}

In this Appendix, an application of the resolution curves is presented. For illustration we assume that a Gaussian transmittance is being used in conjunction with an ideal lens of focal length $f$. The relevant parameters are given as follows:

$$
\begin{array}{rlrl}
f & =10 \mathrm{~cm}, & l=20 \mathrm{~cm}, \\
\lambda & =0.6 \times 10^{-4} \mathrm{~cm}, & \rho=10^{3} \text { points } / \mathrm{cm} . \\
R & =R=4.0 \mathrm{~cm}, & &
\end{array}
$$

It follows from Eq. (19) that

$$
\frac{1}{\Delta \Lambda}=\frac{\lambda l^{\prime}}{2 R} \rho
$$

At this point we will assume that the depth of focus is small enough that $l^{\prime} \cong l_{f}$. This assumption can be checked as a last step in the calculation. Using Eq. (1) together with the above data shows that

$$
l^{\prime} \cong l_{f}^{\prime} \cong 20 \mathrm{~cm}
$$

Assuming the above data, the right-hand side of Eq. (B1) satisfies

$$
\frac{1}{\Delta \Lambda}=0.15
$$

From Fig. 11, the defect parameter $\beta$ consequently is given by

$$
\beta \cong 2.4 \text {. }
$$

The defocus defect $\varepsilon$ can be related, with (14c), to the $\operatorname{defect}$ parameter $\beta$ :

$$
\varepsilon=\left(\frac{\beta}{R}\right)^{2} \lambda / 2 \cong 2.88 \frac{\lambda}{R^{2}} .
$$

Also it is easily shown from Eqs. (1) and (2) that the out-of-focus defect can be written as

$$
\varepsilon=\frac{l_{f}^{\prime}-l^{\prime}}{l_{f}^{\prime} l^{\prime}} \equiv \frac{\Delta l^{\prime}}{l_{f}^{\prime} l^{\prime}} \cong \frac{\Delta l^{\prime}}{l_{f}^{\prime 2}} .
$$

Combining Eqs. (B6) and (B5) leads to an expression for the maximum deviation from focus

$$
\Delta l \cong 2.88 \lambda\left(\frac{l_{f}^{\prime}}{R}\right)^{2}=72 \lambda .
$$

It is easily confirmed that within the range defined by (B7), assumption (B2) is valid. The results indicate that the maximum deviation from the focus is $72 \lambda$ if a line density of $10^{3}$ points $/ \mathrm{cm}$ is to be resolved.

\section{References}

1. M. Born and E. Wolf, Principles of Optics (McGraw-Hill, New York, 1968).

2. T.-C. Poon and M. Motamedi, "Optical/Digital Incoherent Image Processing for Extended Depth of Field," Appl. Opt. 26, 4612 (1987).

3. E. Hecht and A. Zajac, Optics (Addison-Wesley, Reading, MA, 1976).

4. E. H. Linfoot, Fourier Methods in Optical Image Evaluation (Focal Press, London, 1964).

5. E. L. O'Neil, "Transfer Function for an Annular Aperture," J. Opt. Soc. Am. 46, 285 (1956).

6. A. Offner, "Annular Field Systems and the Future of Optical Microlithography," Opt. Eng. 26, 294 (1987).

7. "Comments on 'Diffraction-Free Beams'," available from A. Korpel, U. Iowa.

8. J. W. Goodman, Introduction to Fourier Optics (McGraw-Hill, New York, 1968). 